\title{
Case Report: Evaluating Biomechanical Risk Factors in Carotid Stenosis by Patient-Specific Fluid-Structural Interaction Biomechanical Analysis
}

\author{
Jiaqiu Wang ${ }^{a} b, c$ Jessica Benitez Mendieta ${ }^{b}$ Phani Kumari Paritala ${ }^{b}$ \\ Yuqiao Xiang $^{\text {b }}$ Owen Christopher Raffel ${ }^{b, d, e} \operatorname{Tim~McGahan~}^{f}$ Thomas Lloyd ${ }^{g}$ \\ Zhiyong $\mathrm{Li}^{\mathrm{b}}$, h \\ aSchool of Clinical Sciences, Queensland University of Technology, Brisbane, QLD, Australia; bSchool of Mechanical, \\ Medical and Process Engineering, Queensland University of Technology, Brisbane, QLD, Australia; Institute of \\ Health and Biomedical Innovation (IHBI), Queensland University of Technology, Kelvin Grove, QLD, Australia; \\ ${ }^{\mathrm{d}}$ Department of Cardiology, The Prince Charles Hospital, Brisbane, QLD, Australia; 'School of Medicine, University

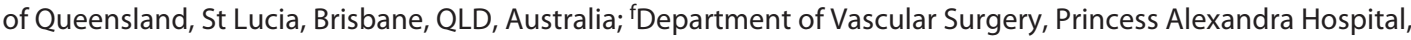 \\ Brisbane, QLD, Australia; ${ }^{9}$ Department of Radiology, Princess Alexandra Hospital Brisbane, Brisbane, QLD, Australia; \\ ${ }^{\mathrm{h}}$ School of Biological Science \& Medical Engineering, Southeast University, Nanjing, China
}

\section{Keywords}

Atherosclerosis · Carotid stenosis · Vulnerable plaque ·

Fluid-structure interaction · Computational fluid dynamics · Biomechanical stress analysis

\footnotetext{
Abstract

Background: Carotid atherosclerosis is one of the main underlying inducements of stroke, which is a leading cause of disability. The morphological feature and biomechanical environment have been found to play important roles in atherosclerotic plaque progression. However, the biomechanics in each patient's blood vessel is complicated and unique. Method: To analyse the biomechanical risk of the patientspecific carotid stenosis, this study used the fluid-structure interaction (FSI) computational biomechanical model. This model coupled both structural and hemodynamic analysis. Two patients with carotid stenosis planned for carotid endarterectomy were included in this study. The 3D models of ca-
}

rotid bifurcation were reconstructed using our in-house-developed protocol based on multisequence magnetic resonance imaging (MRI) data. Patient-specific flow and pressure waveforms were used in the computational analysis. Multiple biomechanical risk factors including structural and hemodynamic stresses were employed in post-processing to assess the plaque vulnerability. Results: Significant difference in morphological and biomechanical conditions between 2 patients was observed. Patient I had a large lipid core and serve stenosis at carotid bulb. The stenosis changed the cross-sectional shape of the lumen. The blood flow pattern changed consequently and led to a complex biomechanical environment. The FSI results suggested a potential plaque progression may lead to a high-risk plaque, if no proper treatment was performed. The patient II had significant tandem stenosis at both common and internal carotid artery (CCA and ICA). From the results of biomechanical factors, both stenoses had a high potential of plaque progression. Especially for the plaque at ICA branch, the current 2 small plaques might fur-

$\begin{aligned} & \text { karger@karger.com } \\ & \text { www.karger.com/ced }\end{aligned}$
Karger ${ }^{\prime /}$


ther enlarge and merge as a large vulnerable plaque. The risk of plaque rupture would also increase. Conclusions: Computational biomechanical analysis is a useful tool to provide the biomechanical risk factors to help clinicians assess and predict the patient-specific plaque vulnerability. The FSI computational model coupling the structural and hemodynamic computational analysis, better replicates the in vivo biomechanical condition, which can provide multiple structural and flow-based risk factors to assess plaque vulnerability.

(c) 2021 S. Karger AG, Basel

\section{Introduction}

Carotid atherosclerosis is one of the main underlying inducements of stroke, which is a leading cause of disability $[1,2]$. The morphological features and hemodynamic environments have been found to play important roles in plaque progression $[3,4]$. At carotid bifurcation, the flow pattern becomes complicated, which is considered as pathogenesis of atherosclerosis $[5,6]$. The biomechanical stress assessment using blood vessel geometrical models has been studied to predict and evaluate the plaque vulnerability [7]. Computational methods have been widely used in the biomechanical stress assessment, from the hemodynamic and structural analysis. The value and distribution of biomechanical forces applied on blood vessels, such as wall shear stress (WSS) and tensile stress can be calculated, which could be used to evaluate the atherosclerotic plaque vulnerability $[8,9]$.

The fluid-structure interaction (FSI) approach couples the computational fluid dynamics (CFD) and structural analysis. The vascular system is a complex FSI system, where the blood flow applies shifty blood pressure on the vessel wall, and the blood flow domain (lumen) is flexible, highly non-linear, and periodically deforming. The deformation of blood vessel with plaques is much more complicated. Therefore, besides the ability of providing both hemodynamic and structural information, the advantage of using FSI model also includes non-uniform pressure load and flexible fluid domain, which can better mimic the realistic vasculature system [10].

Our group has developed the FSI model of the cardiovascular blood vessels on the commercial finite element analysis software platform ANSYS (ANSYS Inc.) [10,11]. Here, we applied the FSI computational modelling strategy to the patient-specific carotid data. The aim of this study was to provide further quantitative assessment for plaque vulnerability on patient-specific cases.

Case Report of Biomechanical Evaluation of Carotid Stenosis

\section{Materials and Methods}

\section{Imaging Data Acquisition and Modelling}

The data used in this study were acquired from the Prince Alexandra Hospital (PAH, Brisbane, QLD, Australia). This study was approved by the Metro South Human Research Ethics Committee (HREC/17/QPAH/181), and patient consent forms were obtained.

Two patients (male, age of 61 and 77 years, respectively) with carotid stenosis planned for carotid endarterectomy were included in this study. Before carotid endarterectomy, carotid bifurcation of each patient was scanned using our established multi-contrast magnetic resonance imaging (MRI) protocol [12]. Four MRI contrast-weighted imaging techniques (including T2 weighted, proton density, T1 weighted, and short T1 inversion recovery) were employed to allow for the identification of the different plaque components. Additionally, 2D electrocardiogram-gated phasecontrast MRI images were acquired to record the massflow profile. The geometric models in this study were reconstructed from MRI data (shown in Fig. 1a, b, d). The image-processing software Amira (version 6.0, Thermo Fisher Scientific) was used for imaging processing, contour segmentation, and $3 \mathrm{D}$ reconstruction.

\section{FSI Model}

FSI analysis was performed on ANSYS Workbench platform (version 19.0, ANSYS Inc.). The fluent CFD and transient structural analysis were fully coupled by the system coupling framework. The lateral surface of lumen was set as the fluid-structure interface. For both models, the timestep was set as $0.01 \mathrm{~s}$, which was determined by the CFD timestep-independent check and conjunct with the FSI convergence ability.

In the CFD participant, the fluid domain was meshed with tetrahedral elements with inflation layers. The smoothing and remeshing methods were given to the dynamic fluid domain. Blood flow was assumed as incompressible laminar and Newtonian flow. The viscosity and density of blood were given as $0.00345 \mathrm{~Pa}$ and 1,050 $\mathrm{kg} / \mathrm{m}^{3}$, respectively [13]. Patient-specific time-dependent massflow rate acquired from phase-contrast MRI data was set as inlet boundary condition. Based on the patient-specific flowrate waveform, the pressure profile was scaled into the range within the high/low value of patient's pressure measurement (shown in Fig. 1c, e). To avoid the unstable results in the first several timesteps, an extension of 10 timesteps was added to the original profiles.

In the structural participant, the geometries were meshed by using automatic proximity and curvature size function. The linear elastic material properties were given to the arterial wall (Young's modulus, 0.6 MPa; Poisson's ratio, 0.48) and lipid (Young's modulus, $0.02 \mathrm{MPa}$; Poisson's ratio, 0.48). The side edges on the common, internal, and external carotid artery (CCA, ICA, and ECA, respectively) were given fixed supports.

\section{Analysis of Results}

The post-processing software ANSYS CFD-post (version 19.0, ANSYS Inc.), Tecplot (Tecplot 360 EX 2015 R2, Tecplot Inc.), and the data analysis software Origin (version 2018, OriginLab Corp.) were used for result analysis and visualization. In the CFD participant, WSS is the most commonly used index to describe the hemodynamic behaviour. The area with low WSS $(<1 \mathrm{~Pa})$ is associated with a disturbed flow and indicates an atherosclerosis-prone area, while the area with high value $(>3 \mathrm{~Pa})$ induces the behaviour change of the endothelial cell and promotes the high-risk plaques 


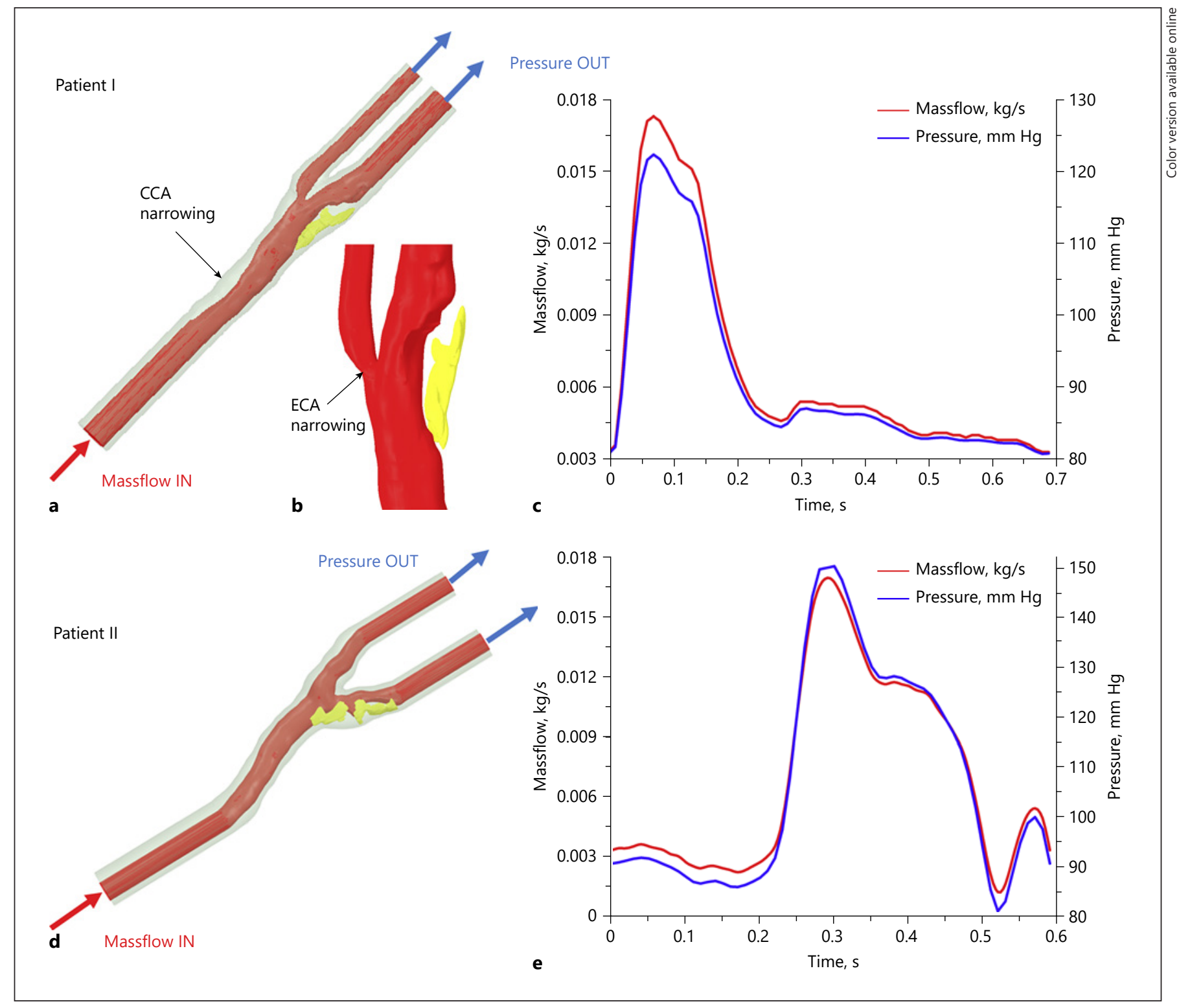

Fig. 1. Patient-specific geometric models and boundary conditions (top, patient I; bottom, patient II), patientspecific carotid bifurcation model with extended inlet and outlets $(\mathbf{a}, \mathbf{d})$; the zoom view of the lipid at carotid bulb location (b); the flow and pressure profiles (c, e). ECA, external carotid artery; CCA, common carotid artery.

$[14,15]$. Besides, there are several WSS-derived risk factors associated to the atherosclerosis, that is, time-averaged WSS (TaWSS), oscillatory shear index (OSI), and relative residence time (RRT). OSI describes the difference between WSS acting in directions and the direction of temporal mean WSS vector [16]. RRT is marked by low WSS magnitude and high oscillatory WSS [17]. Normally, a low TaWSS, high OSI, and RRT are used as indicators of the atherogenesis region $[18,19]$. In the structural analysis participant, the maximum principle stress (Stress-P1) was analysed. The high stress area on the fibrous cap was presumed as a rupture-prone vulnerable area $[20,21]$.

\section{Results}

\section{Investigation of Patient I}

This patient had a large lipid core at the carotid bulb location. The stenosis ratio was calculated as $72 \%$ (based on the standard of European Carotid Surgery Trial [ECST] [22]). Another narrowing occurred at the ECA branch near the bifurcation apex, a slight stenosis was found at CCA, which could be visualized in Figure 1a, b. 


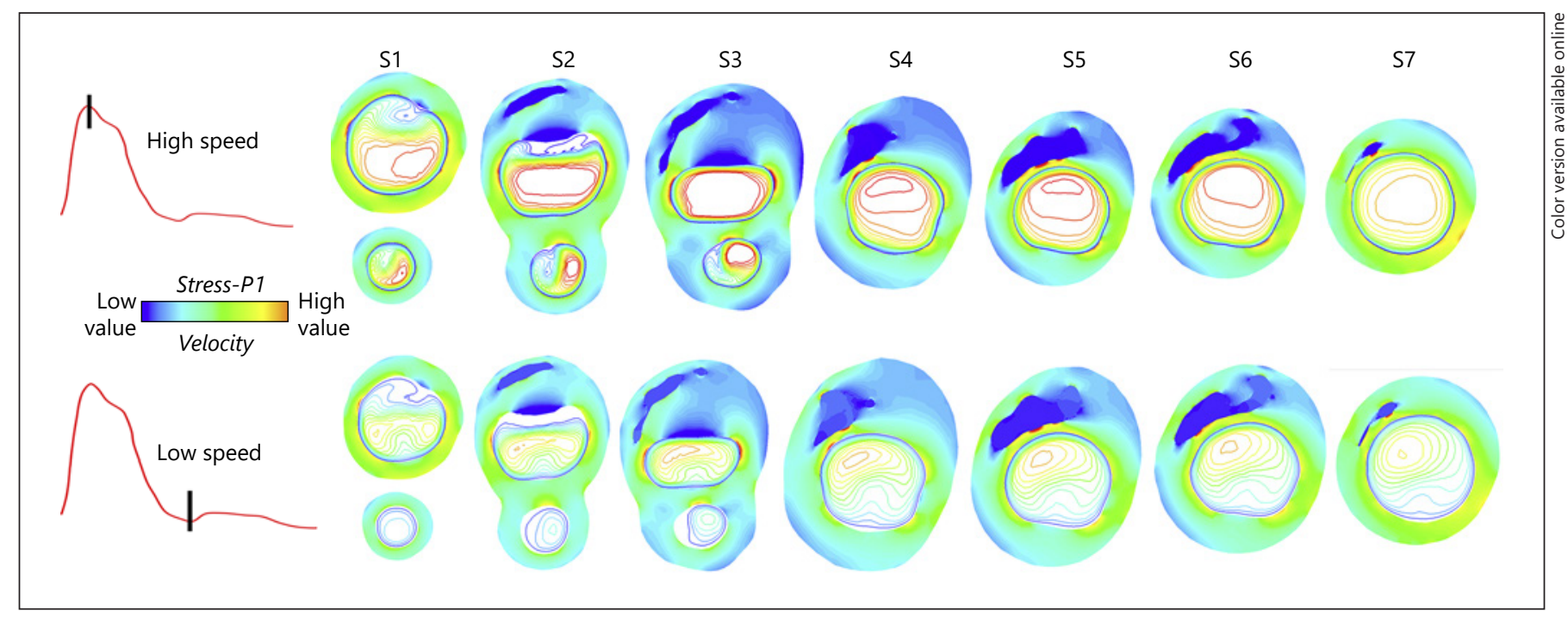

Fig. 2. Maximum principle stress (Stress-P1) and velocity pattern in selected planes from patient I, at both timesteps with high and low massflow. The dark blue shows the lipid plaque.

In Figure 2, the Stress-P1 and velocity pattern were plotted in selected planes. By comparing the results in the same position but under different high/low massflow rates, the distribution pattern of Stress-P1 at the high massflow was similar as that at the low massflow, and the magnitude at the corresponding position was a bit higher than that at the low-speed timestep. The flow patterns were significantly different between the high- and lowspeed timesteps. The irregular shape of flow pattern suggested that if the flow distribution was complicated, low and oscillatory WSS might happen. Comparing the flow patterns in different locations, the flow became complex after passing the carotid bifurcation. It suggested the carotid bifurcation was high-risk area of developing atherosclerosis. At the structural analysis participant, the StressP1 indicated the position with high stress, which might cause structure failure. The high stress could be found near the plaque area where the fibrous cap was thin. Also, the high stress was found at the near-lumen area where the curvature was large and the lumen shape was sharp.

WSS distribution was plotted in Figure 3. WSS is highly related to the flow velocity and flow domain area. Generally, a higher WSS area could be found at the high-speed timestep and vice versa. In detail, when the WSS was at the high-speed timestep, the high WSS was found at the stenotic location. These areas had risks of endothelial damage. Downstream from the stenosis, at the low-speed timestep, a low WSS area was found mostly near the ste- nosis. As the low WSS area was the atherosclerosis-prone location, when low WSS was detected downstream of the stenosis, the plaque might further develop downstream following the low WSS direction.

The WSS at a high-/low-speed timestep only reflected the WSS distribution at a single timestep and was not enough to evaluate the flow behaviour in carotid bifurcation. As illustrated, TaWSS, OSI, and RRT are the WSSderived parameters calculated from all the timesteps. In Figure $3 c$, the TaWSS was plotted, showing that a high value was found at the significant narrowing at the ECA branch. The low value of TaWSS (shown in Fig. 3d) was clearly found at the downstream of stenotic locations, in the potential atherosclerosis-prone area. The high values of OSI (Fig. 3e) and RRT (Fig. 3f) were both found at the downstream of small CCA stenosis and carotid bulb stenosis (ICA plaque area). This also proved there was risk of development of atherosclerosis.

This patient was determined as medium-risk based on clinical experience; however, from the biomechanical risk factors, the potential risk area was found and might further result in a high-risk plaque if no proper treatment was performed. At the near-lipid plaque area, a high structural stress concentration was found, which might cause plaque rupture. Based on the hemodynamic analysis, the atherosclerosis-prone area was found near the current stenosis. It was a potential risk of further developing the current plaque and becoming a high-risk plaque. 


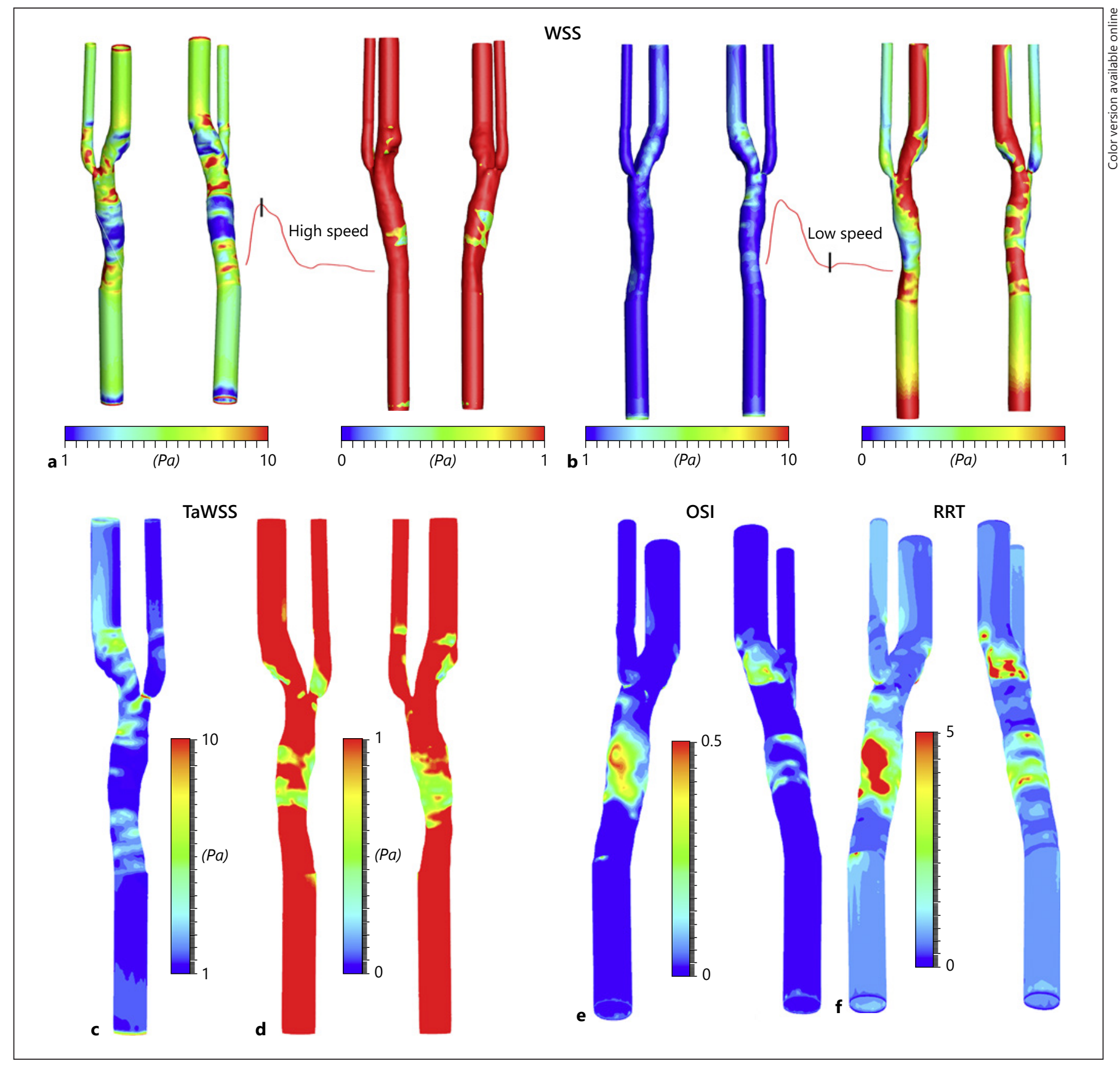

Fig. 3. The plot of WSS and its derived parameters of patient I. At the timestep with high flow velocity (a) and the timestep with low flow velocity (b), the WSS was plotted in a normal WSS range scale and low WSS range scale $(<1 \mathrm{~Pa})$. In the plot with a low WSS range scale, the low WSS regions are easy to locate. The WSS-derived parameters included TaWSS, plotted in both normal range (c) and low value range (d) $(<1 \mathrm{~Pa})$, OSI (e), and RRT (f). WSS, wall shear stress; TaWSS, time-averaged WSS; OSI, oscillatory shear index; RRT, relative residence time. 


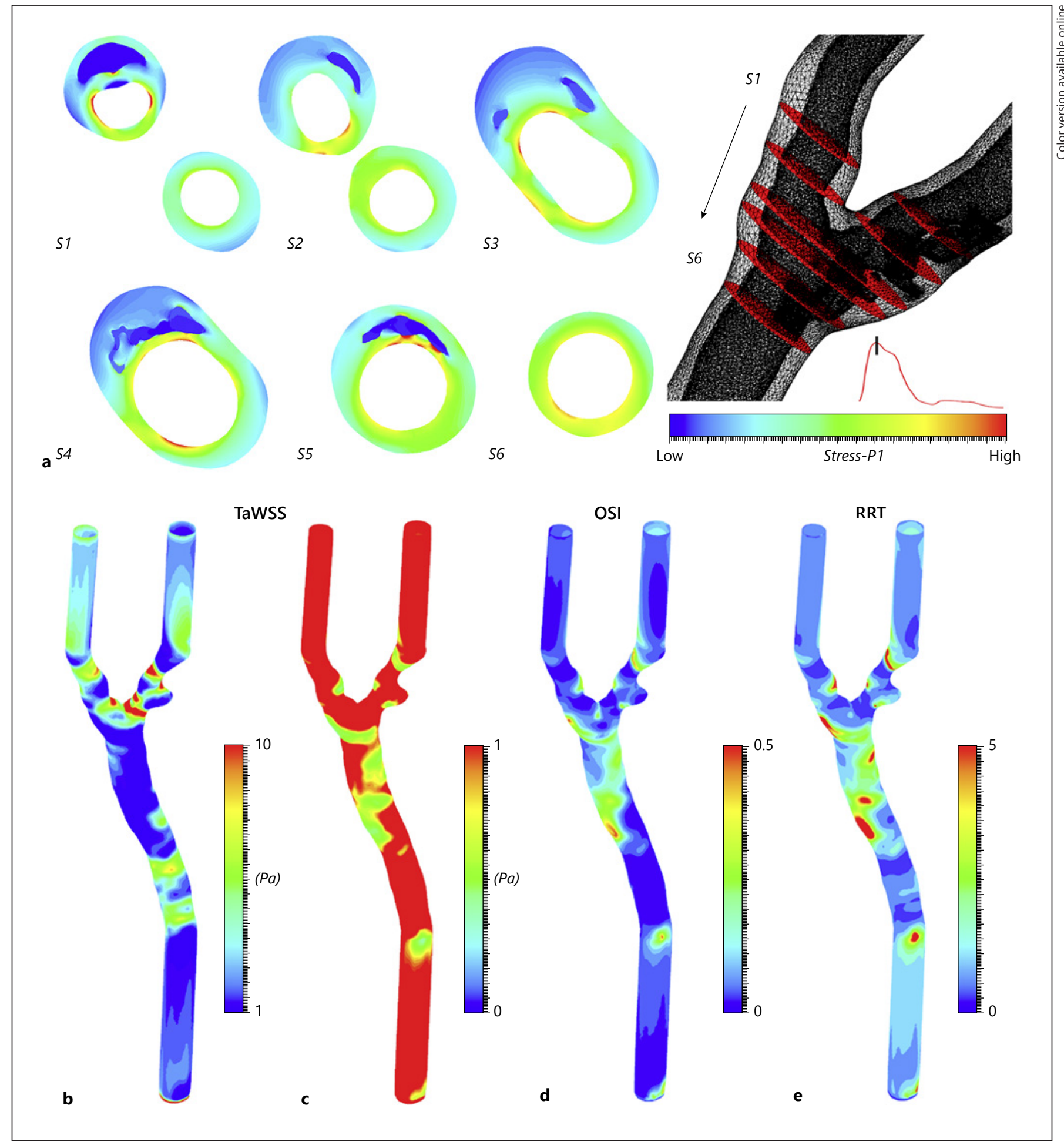

Fig. 4. Plots of biomechanical risk factors from patient II. The plot of structural stress in 6 planes from the 3D reconstructed carotid bifurcation of patient II at the high-speed timestep (a); TaWSS plot in the normal value range (b); TaWSS plot in the low value range $(\mathbf{c})(<1 \mathrm{~Pa})$; OSI (d); and RRT (e). TaWSS, time-averaged wall shear stress; OSI, oscillatory shear index; RRT, relative residence time. 


\section{Investigation of Patient II}

Patient II previously has been studied by using CFDonly presumptive models [19]. This patient had severe stenosis in both CCA and ICA, where the ICA stenosis consisted of 2 small stenoses and had complex partitions of plaque. The stenosis ratio determined by ECST standard was $76 \%$. The large lipid plaque was found having intruded into the lumen (shown in Fig. 1d).

From the structural analysis, the structural stress indicated the high-risk location of a structural failure. In Figure 4a, Stress-P1 was plotted in 6 planes. At the S6, there was no plaque and the stress was in a normal range. At the other slices, high stress areas were found at the proximity of plaque, especially near the thin fibrous cap area, like S4 and S5.

Further on our previous CFD-only model, the TaWSS, OSI, and RRT were plotted in Figure $4 \mathrm{~b}-\mathrm{e}$. These plots illustrated the potential atherosclerosis-prone area. Firstly, after the stenosis at CCA, the blood flow caused the low TaWSS, high OSI, and RRT area and may further cause the current plaque to develop. When the flow passed the bifurcation, at ICA, the atherosclerosis-prone area was detected between the 2 small stenoses; this area had potential risk of forming a new atherosclerotic plaque and connecting the 2 current plaques. If a large plaque formed, the risk of clinical event would be higher than current medium risk. Also, in the ECA branch, there was a risk of new atherosclerosis development.

Following our previous CFD-only study [19], we further introduced the FSI model to study this patient case. In the FSI model, the finding from the previous CFDonly model was reiterated; the current plaque might further develop if the stenosis was not removed. Also, there was a potential of new atherosclerosis development at the ECA branch. This patient had a complex blood vessel shape near the bifurcation, which increased the risk of vulnerable plaque formation.

\section{Discussion}

\section{Advantage of This Study}

In this study, the FSI model was applied to the patientspecific carotid bifurcations. Compared to the CFD-only model, the FSI model could help find the potential risk of clinical cardiovascular disease events by providing the quantitative biomechanical risk factors in both structural and hemodynamic analyses. Furthermore, as suggested in a previous study [10], the flexible vessel wall makes the WSS result more convincing. By comparing with the conclusion from a previous CFD-only study [19], there were some differences found on the values of hemodynamic parameters. But it was noticed the pattern of the abnormal area with low WSS, high OSI, and RRT was similar in both FSI and CFD-only models, which proved the CFD-only model could be an effective alternative if only hemodynamic factors were required.

Some other technique advantages include, firstly, the carotid bifurcation model was reconstructed based on a novel multi-sequence MRI segmentation protocol, which was more precise. Secondly, the use of patient-specific boundary conditions was an improvement compared to the model using a universal profile. Lastly, in this study, we utilized the patient-specific case study. Based on the biomechanical risk factors, the plaque vulnerability was evaluated, and the potential progression of atherosclerosis was predicted. These results from biomechanical analysis may act as additional factors to help clinicians in risk assessment of patient vulnerability and treatment plan.

\section{Study Limitations}

This study has several limitations which required further investigation. Firstly, the current FSI model required huge time and computational consumption. And the convergence stability of the FSI model was still a challenge. These technique limitations hindered the computational biomechanical analysis from being widely used in clinical practice. Secondly, the patient-specific tissue elasticity profile is still not available for in vivo measurement. The realistic material properties are highly nonlinear and inhomogenous, also with wide variation between individuals. The use of simplified elasticity may have a big influence on the analysis results. Finally, we have to say that the relationship between the clinical event and biomechanical risk factors is still unclear. The criteria threshold values of these biomechanical risk factors are not available yet, and further studies on larger scale patient populations are needed to determine the critical values before this method is translated to clinical practice.

\section{Conclusion}

In this study, 2 patient cases with carotid atherosclerosis were studied. Based on the biomechanical risk factors, plaque vulnerability was evaluated, and the potential progression of atherosclerosis was predicted. The FSI model provided both structural and hemodynamic analyses and could mimic the flexible vessel wall. However, the complexity and computational cost of the FSI model are still a challenge. 


\section{Acknowledgement}

The authors would like to thank the team at Princess Alexandra Hospital (Brisbane, Australia) for identifying potential research participants, obtaining patient consent, and providing facilities, especially Gillian Jagger.

\section{Statement of Ethics}

Written informed consent was obtained from the patients for publication of their case reports and any accompanying images. This study was approved by the Metro South Human Research Ethics Committee (HREC/17/QPAH/181).

\section{Conflict of Interest Statement}

The authors declare that they have no conflict of interest.

\section{Funding Sources}

The authors gratefully acknowledge financial support from the Australian Research Council (ARC DP200103492 and DP200101970), the National Natural Science Foundation of China (Grant Nos. 11972118, 11772093, and 61821002), the PA Research Foundation (PARF), and the Prince Charles Hospital Foundation (TPCH Foundation NI2019-19).

\section{Author Contributions}

J.W., J.M., P.P., Y.X., O.R., and Z.L. contributed to the conception and design of the study, result discussion, and manuscript draft. T.M. and T.L. organized the data and sample collection. P.P. conducted the staining histology. J.M. performed the MRI processing and 3D modelling. J.W. carried out the computational simulation, data post-processing, and analysis. All authors approved the final version of the manuscript.

\section{References}

1 Bertoletti G, Varroni A, Misuraca M, Massucci M, Pacelli A, Ciacciarelli M, et al. Carotid artery diameters, carotid endarterectomy techniques and restenosis. J Vasc Med Surg. 2013;1(2):1-5.

2 Benjamin EJ, Muntner P, Alonso A, Bittencourt MS, Callaway CW, Carson AP, et al. Heart disease and stroke statistics-2019 update: a report from the American heart association. Circulation. 2019 Mar 5;139(10):e56e528.

3 Lusby RJ, Woodcock JP, MacHleder HI, Ferrell LD, Jeans WD, Skidmore R, et al. Transient ischaemic attacks: the static and dynamic morphology of the carotid artery bifurcation. Br J Surg. 1982;69 Suppl:S41-4.

4 Malek AM, Alper SL, Izumo S. Hemodynamic shear stress and its role in atherosclerosis. JAMA. 1999 Dec;282(21):2035.

$5 \mathrm{Xu} \mathrm{XY,} \mathrm{Collins} \mathrm{MW.} \mathrm{A} \mathrm{review} \mathrm{of} \mathrm{the} \mathrm{numeri-}$ cal analysis of blood flow in arterial bifurcations. Proc Inst Mech Eng H. 1990;204(4): 205-16.

6 Bijari PB, Antiga L, Gallo D, Wasserman BA, Steinman DA. Improved prediction of disturbed flow via hemodynamically-inspired geometric variables. J Biomech. 2012;45(9): $1632-7$.

7 Tang D, Kamm RD, Yang C, Zheng J, Canton G, Bach R, et al. Image-based modeling for better understanding and assessment of atherosclerotic plaque progression and vulnerability: data, modeling, validation, uncertainty and predictions. J Biomech. 2014 Mar;47(4): $834-46$.

8 Kwak BR, Bäck M, Bochaton-Piallat ML, Caligiuri G, Daemen MJ, Davies PF, et al. Biomechanical factors in atherosclerosis: mechanisms and clinical implications. Eur Heart J. 2014;35(43):3013-20d.
9 Morbiducci U, Kok AM, Kwak BR, Stone PH, Steinman DA, Wentzel JJ. Atherosclerosis at arterial bifurcations: evidence for the role of haemodynamics and geometry. Thromb Haemost. 2016 Mar;115(3):484-92.

10 Wang J, Paritala PK, Mendieta JB, Komori Y, Raffel OC, Gu Y, et al. Optical coherence tomography-based patient-specific coronary artery reconstruction and fluid-structure interaction simulation. Biomech Model Mechanobiol. 2020 Feb;19(1):7-20.

11 Chimakurthi SK, Reuss S, Tooley M, Scampoli S. ANSYS workbench system coupling: a state-of-the-art computational framework for analyzing multiphysics problems. Eng Comput. 2018;34(2):385-411.

12 Benitez J, Fontanarosa D, Wang J, Paritala PK, McGahan T, Lloyd T, et al. Evaluating the impact of calcification on plaque vulnerability from the aspect of mechanical interaction between blood flow and artery based on MRI. Ann Biomed Eng. 2020 Oct.

13 Liu B, Tang D. Influence of non-Newtonian properties of blood on the wall shear stress in human atherosclerotic right coronary arteries. Mol Cell Biomech. 2011;8(1):73-90.

14 Dolan JM, Kolega J, Meng H. High wall shear stress and spatial gradients in vascular pathology: a review. Ann Biomed Eng. 2013;41(7): 1411-27.

15 Eshtehardi P, Brown AJ, Bhargava A, Costopoulos C, Hung OY, Corban MT, et al. High wall shear stress and high-risk plaque: an emerging concept. Int J Cardiovasc Imaging. 2017;33(7):1089-99.
$16 \mathrm{Ku}$ DN, Giddens DP, Zarins CK, Glagov S Pulsatile flow and atherosclerosis in the human carotid bifurcation. Positive correlation between plaque location and low oscillating shear stress. Arteriosclerosis. 1985;5(3):293302

17 Himburg HA, Grzybowski DM, Hazel AL, LaMack JA, Li XM, Friedman MH. Spatial comparison between wall shear stress measures and porcine arterial endothelial permeability. Am J Physiol Heart Circ Physiol. 2004; 286(5):H1916-22.

18 Soulis JV, Lampri OP, Fytanidis DK, Giannoglou GD. Relative residence time and oscillatory shear index of non-Newtonian flow models in aorta. 10th Int Work Biomed Eng Bioeng. 2011(1):1-4.

19 Wang J, Paritala PK, Mendieta JB, Gu Y, Raffel OC, McGahan T, et al. Carotid bifurcation with tandem stenosis-a patient-specific case study combined in vivo imaging, in vitro histology and in silico simulation. Front Bioeng Biotechnol. 2019 Nov;7(November):111.

20 Paritala PK, Yarlagadda PKDV, Wang J, Gu $\mathrm{Y}, \mathrm{Li} \mathrm{Z}$. Numerical investigation of atherosclerotic plaque rupture using optical coherence tomography imaging and XFEM. Eng Fract Mech. 2018;204(November):531-41.

21 Paritala PK, Yarlagadda T, Wang J, Gu YT, Li Z. Prediction of atherosclerotic plaque life: perceptions from fatigue analysis. Procedia Manuf. 2019;30:522-9.

22 European Carotid Surgery Trialists' Collaborative Group. Randomised trial of endarterectomy for recently symptomatic carotid stenosis: final results of the MRC European carotid surgery trial (ECST). Lancet. 1998 May; 351(9113):1379-87. 